\title{
ORIENTATION OF METASTABLE EL2 UNDER UNIAXIAL STRESS
}

\author{
A. Babiński and A. WYSMOLEK \\ Institute of Experimental Physics, Warsaw University \\ Hoża 69, 00-681 Warszawa, Poland
}

\begin{abstract}
Results of electrical resistivity and Hall measurements of $n$-type GaAs under uniaxial stress along [111] direction performed at low temperature are presented. After the transformation of the EL2 defect into its metastable configuration, a stress-induced increase in electrical resistivity related to the capture of electrons by the acceptor state of the metastable EL2 ([EL2* ${ }^{-10}$ ) was observed. It was found that the stress-induced increase in resistivity depended on the method of EL2-photoquenching. The observed effects are explained as the reorientation of EL2* centers in the crystal. The stress coefficients of the triple degenerate and the single degenerate sublevels of the $\left[\mathrm{EL} 2^{*}\right]^{-10}$ are found to be equal to $-17 \mathrm{meV} / \mathrm{GPa}$ and $-41 \mathrm{meV} / \mathrm{GPa}$.

PACS numbers: 71.55.Eq, 72.20.Fr, 72.80.Ey
\end{abstract}

The recent measurements of the thermal recovery of the EL2-related absorption performed under uniaxial stress [1] have shown the trigonal $C_{3 v}$ symmetry of the metastable EL2 defect ([EL2*]). It was found moreover that orientation of EL2* centers depended on the method of the EL2-photoquenching [2] which confirmed the attribution of EL2 metastability to the transformation $\mathrm{As}_{\mathrm{Ga}} \leftrightarrow \mathrm{V}_{\mathrm{Ga}} \mathrm{As}_{\mathrm{i}}$ $[3,4]$. The key role in the thermal recovery of EL2 in $n$-type GaAs is played by the acceptor level of EL2* $\left(\left[\mathrm{EL} 2^{*}\right]^{-10}\right)[5,6]$ which is orientationally degenerate and split under $[111]$ stress into two components: the triplet $\left(\left[\mathrm{EL} 2^{*}\right]_{\mathrm{T}}^{-10}\right)$ and the singlet $\left(\left[\mathrm{EL} 2^{*}\right]_{\mathrm{S}}^{-10}\right)$ sublevels. We performed the electrical conductivity and Hall effect measurements of $n$-type GaAs in order to investigate the electrical properties of these levels under [111] uniaxial stress. Measurements were done at low temperatures $(4.2 \mathrm{~K} . .30 \mathrm{~K})$ with an access of light.

The investigated material was $n$-type liquid encapsulated Czochralski (LEC) GaAs $\left(n=3.6 \times 10^{16} \mathrm{~cm}^{-3}\right)$ with EL2 concentration equal to $4.5 \times 10^{15} \mathrm{~cm}^{-3}$. The [111]-oriented sample was placed in an optical cryostat and uniaxial stress up to $700 \mathrm{MPa}$ was obtained with a press spring apparatus. The electrical resistivity and Hall effect in the van der Pauw configuration were measured.

We found that while EL2 was in its normal configuration (just after the sample was cooled down in the dark) the sample resistivity very weakly depended on applied stress (see open circles in Fig. 1). After the transformation of EL2 


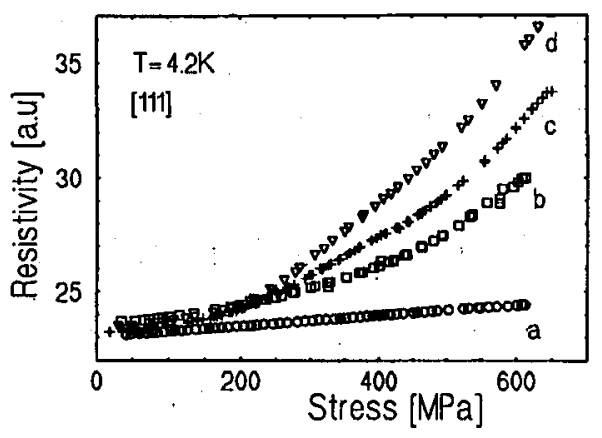

Fig. 1. The dependence of a sample resistivity on stress along [111] direction measured at $T=4.2 \mathrm{~K}: a-$ just after cooling down the sample in the dark, $b$ - after EL2-photoquenching performed under high stress $(600 \mathrm{MPa}), c$ - after EL2-photoquenching performed partially under high stress and partially without stress, $d$ - after EL2-photoquenching performed without external stress.

into the metastable configuration with $1.05 \mu \mathrm{m}$ light the stress induced increase in the sample resistivity appeared. The observed stress-induced increase in resistivity was due to the capture of electrons by the both $\left[E L 2^{*}\right]^{-10}$ split sublevels which move downwards with respect to the conduction band minimum when the stress is applied [7].

It was observed that the amplitude of the stress-induced resistivity increase depended on the method of EL2-photoquenching. The effect was bigger if EL2-photoquenching was performed without external stress and lower if high stress (600 MPa) was applied during illumination (see Fig. 1). It was observed that after sufficiently long illumination performed at high stress the subsequent illumination without stress did not change the stress-dependence of resistivity. However, if EL2-photoquenching under high stress was not completed, then additional illumination without stress caused further changes in the stress-dependence of resistivity (see Fig. 1). This result means that after sufficiently long illumination performed under high stress all EL2 centers were in the metastable configuration and the ratio of centers created along and aslant the axis of stress (related to the singlet and triplet sublevels of split [EL2*]-10 level) could not be changed during an experiment as long as the temperature was sufficiently low (and thermal recovery was not possible).

The observed effect was due to the dependence of EL2* center orientation in the crystal on the method of EL2-photoquenching. If no stress is applied to the sample, EL2-photoquenching with unpolarized light creates EL2* centers along all four [111] crystallographic directions. As a result, the resistivity changes observed under stress are due to the capture of electrons by both split [EL2*]-10 sublevels: the singlet $\left[\mathrm{EL} 2^{*}\right]_{\mathrm{S}}^{-10}$ related to centers oriented along the axis of stress and the triplet $\left[\mathrm{EL} 2^{*}\right]_{\mathrm{T}}^{-10}$ related to centers oriented aslant the axis of stress. If EL2-photoquenching is performed under high [111] stress, the pressure-induced 
optical recovery $[8]$ becomes effective for the $\left[E L 2^{*}\right]^{-10}$ sublevel, which is deeper in a forbidden gap. As a result, after sufficiently long time of such an illumination mainly EL2* centers related to the sublevel of higher energy are present in the crystal. From the optical measurements of Trautman [2] it is clear that such a situation can be created either by illumination of a stressed sample with white unpolarized light (like in our experiment) or by illumination of an unstressed sample, but performed with light polarized perpendicularly to the direction of stress applied afterwards (i.e. after EL2-photoquenching). It means that centers present in our sample after EL2-photoquenching performed under a high stress were oriented aslant the axis of stress and the triplet $\left[\mathrm{EL} 2^{*}\right]_{\mathrm{T}}^{-10}$ level was of higher energy than the singlet one.

Our results are consistent only with the isolated $\mathrm{As}_{\mathrm{Ga}}$ model of the EL2 center. In that model the transition to the metastable configuration is connected with the transition of antisite $\mathrm{As}_{\mathrm{Ga}}$ atom into the interstitial position on the [111] direction. In the $\mathrm{As}_{\mathrm{Ga}}-\mathrm{As}_{\mathrm{i}}$ defect pair model [9] the orientation of EL2* centers results from the orientation of $\mathrm{As}_{\mathrm{Ga}}-\mathrm{As}_{\mathrm{i}}$ defect pair. The reorientation of such a pair would have to result not only from the transition of the $\mathrm{As}_{\mathrm{Ga}}$ atom into the interstitial position but it would involve the reorientation of the $A s_{i}$ atom as well.

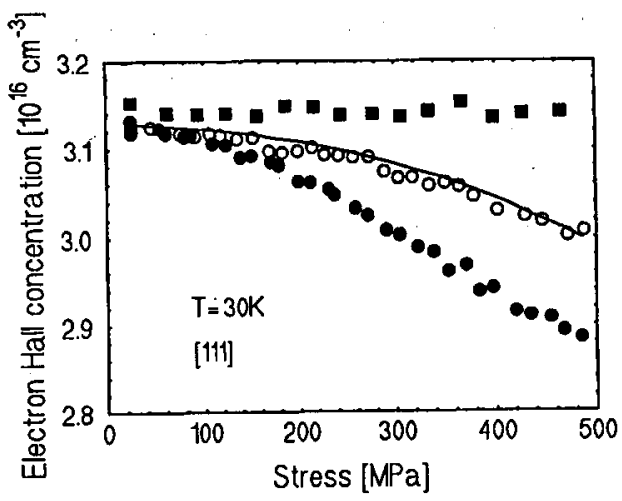

Fig. 2. The electron Hall concentration under uniaxial stress along [111] direction measured at $T=30 \mathrm{~K}$ just after cooling down the sample in the dark (full squares), after EL2-photoquenching performed under stress of $500 \mathrm{MPa}$ (open circles) and after EL2-photoquenching performed without external stress (full circles). The theoretical dependence of electron concentration after EL2-photoquenching performed under high stress (continuous line) was obtained with the following parameters: $E_{0}=14 \mathrm{meV}$ and $\mathrm{d} E_{\mathrm{T}} / \mathrm{d} \chi_{[111]}=-17 \mathrm{meV} / \mathrm{GPa}$.

The results of Hall concentration measurements done at $T=30 \mathrm{~K}$ are presented in Fig. 2. We performed the numerical analysis of the obtained stress-dependences of electron concentration at $T=30 \mathrm{~K}$ assuming the energy of the unsplit $\left[\mathrm{EL} 2^{*}\right]^{-/ 0}$ level $\left(E_{0}=14 \mathrm{meV}\right)$ from Ref. [5]. The best fit to experimental points measured after EL2-photoquenching performed under high stress, when only the $\left[\mathrm{EL} 2^{*}\right]_{\mathrm{T}}^{-10}$ sublevel is present (all EL2* centers are oriented aslant the axis of 
stress), was obtained with $\mathrm{d} E_{\mathrm{T}} / \mathrm{d} \chi_{[111]}=-17 \mathrm{meV} / \mathrm{GPa}$ (see continuous line in Fig. 2). Taking into account the hydrostatic pressure coefficient of the $\left[E L 2^{*}\right]^{-10}$ level $(-23 \mathrm{meV} / \mathrm{GPa} \text { from [5]) we can find the stress coefficient of the [EL2* }]_{\mathrm{S}}^{-10}$ sublevel to be equal to $\mathrm{d} E_{\mathrm{S}} / \mathrm{d} \chi_{[111]}=-41 \mathrm{meV} / \mathrm{GPa}$. For the detailed results of numerical analysis see Ref. [10].

In conclusion, it should be emphasized that our results are fully consistent with the trigonal $C_{3 v}$ symmetry of the metastable EL2 and the observed dependence of stress-induced resistivity under [111] stress on the method of EL2-photoquenching strongly supports the isolated $\mathrm{As}_{\mathrm{Ga}}$ model of the EL2 center.

\section{References}

[1] P. Trautman, J.M. Baranowski, Phys. Rev. Lett. 69, 664 (1992).

[2] P. Trautman, J.M. Baranowski, A. Babiński, Mater. Sci. Forum 143-147, 1007 (1994).

[3] J.M. Baranowski, M. Kamińska, W. Kuszko, P. Walczak, P. Trautman, M. Jeżewski, Mater. Sci. Forum 10-12, 19 (1986).

[4] J. Dąbrowski, M. Scheffler, Phys. Rev. Lett. 60, 2183 (1988).

[5] M. Baj, P. Dreszer, A. Babiński, Phys. Rev. B 43, 2070 (1991).

[6] P. Dreszer, M. Baj, J. Appl. Phys. 70, 2679 (1991).

[7] A. Babiński, A. Wysmołek, M. Baj, Mater. Sci. Forum 143-147, 1051 (1994).

[8] M. Baj, P. Dreszer, Phys. Rev. B 39, 10470 (1989).

[9] B.K. Meyer, D.M. Hofmann, J.R. Niklas, J.-M. Spaeth, Phys. Rev. B 36, 1332 (1987).

[10] A. Babiński, A. Wysmolek, J.M. Baranowski, Phys. Rev. B 50, 10656 (1994). 